\title{
Triple integral relations involving certain special functions
}

\author{
V.B.L. Chaurasia* and R.C. Meghwal** \\ *Department of Mathematics, University of Rajasthan, Jaipur-302004, India \\ **Department of Mathematics, Government Post Graduate College, Neemuch-458441, India
}

\begin{abstract}
The main aim of this paper is to obtain new triple integral relations that involve $\overline{\mathrm{H}}$-function and the multivariable H-function. The main results of our paper are unified in nature and capable of yielding several cases of interests (New and known).
\end{abstract}

Key Words and Phrases: $\overline{\mathrm{H}}$-function, H-function, Multivariable H-function

\section{Introduction}

The $\overline{\mathrm{H}}$-function [2] occurring in this paper will be defined and represented in the following manner

$$
\begin{array}{r}
\bar{H}_{p, q}^{m, n}[z]=\bar{H}_{p, q}^{m, n}\left[z \mid \begin{array}{l}
\left(a_{j}, \alpha_{j} ; A_{j}\right)_{1, n},\left(a_{j}, \alpha_{j}\right)_{n+1, p} \\
\left(b_{j}, \beta_{j}\right)_{1, m},\left(b_{j}, \beta_{j}, B_{j}\right)_{m+1, q}
\end{array}\right] \\
=\frac{1}{(2 \pi \omega)} \int_{-\omega \infty}^{+\omega \infty} \theta(s) z^{s} d s, \quad \text { where } \\
\theta(s)=\frac{\prod_{j=1}^{m} \Gamma\left(b_{j}-\beta_{j} s\right) \prod_{j=1}^{n} \Gamma\left(1-a_{j}+\alpha_{j} s\right)}{\prod_{j=m+1}^{A_{j}} \Gamma\left(1-b_{j}+\beta_{j} s\right)} \prod_{j=n+1}^{p} \Gamma\left(a_{j}-\alpha_{j} s\right)
\end{array}
$$

For details about this function we may refer to [2].

The multivariable $\mathrm{H}$-function due to Srivastava and Panda [5] is defined and represented as follows

2000 Mathematics Subject Classification: 30C45, 33C60, 33C65

$$
\begin{aligned}
& \mathrm{H}\left[\mathrm{z}_{1}, \ldots, \mathrm{z}_{\mathrm{r}}\right]=\underset{\mathrm{A}, \mathrm{C}:\left[\mathrm{B}^{\prime}, \mathrm{D}\right] ; \ldots ;\left[\mathrm{B}^{(\mathrm{r})}, \mathrm{D}^{(\mathrm{r})}\right]}{0, \lambda:\left(\mathrm{U}^{\prime}, \mathrm{V}^{\prime}\right) ; \ldots ;\left(\mathrm{U}^{(\mathrm{r})}, \mathrm{V}^{(\mathrm{r})}\right)}\left[\begin{array}{l}
{\left[(\mathrm{a}): \theta^{\prime}, \ldots, \theta^{(\mathrm{r})}\right]:\left[\mathrm{b}^{\prime}: \phi^{\prime}\right] ; \ldots ;\left[\mathrm{b}^{(\mathrm{r})}: \phi^{(\mathrm{r})}\right] ;} \\
{\left[(\mathrm{c}): \psi^{\prime}, \ldots, \psi^{(\mathrm{r})}\right]:\left[\mathrm{d}: \delta^{\prime}\right] ; \ldots ;\left[\mathrm{d}^{(\mathrm{r})}: \delta^{(\mathrm{r})}\right] ;}
\end{array} \mathrm{z}_{1}, \ldots, \mathrm{z}_{\mathrm{r}}\right]
\end{aligned}
$$

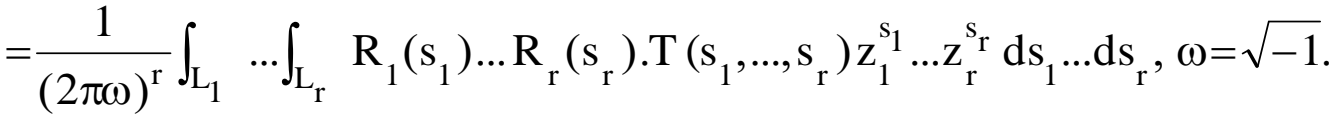

where

$$
R_{i} s_{i}=\frac{\prod_{j=1}^{U^{(i)}} \Gamma\left(d_{j}^{(i)}-\delta_{j}^{(i)} s_{i}\right) \prod_{j=1}^{V^{(i)}} \Gamma\left(1-b_{j}^{(i)}+\phi_{j}^{(i)} s_{i}\right)}{\prod_{j=u^{(i)}+1}^{D^{(i)}} \Gamma\left(1-d_{j}^{(i)}+\delta_{j}^{(i)} s_{i}\right) \prod_{j=v^{(i)}+1}^{B^{(i)}} \Gamma\left(b_{j}^{(i)}-\phi_{j}^{(i)} s_{i}\right)}, \forall i=1, \ldots, r
$$




$$
\mathrm{T}\left(\mathrm{s}_{1}, \ldots, \mathrm{s}_{\mathrm{r}}\right)=\frac{\prod_{\mathrm{j}=1}^{\lambda} \Gamma\left(1-\mathrm{a}_{j}+\sum_{\mathrm{i}=1}^{\mathrm{r}} \theta_{j}^{(\mathrm{i})} \mathrm{s}_{\mathrm{i}}\right)}{\prod_{\mathrm{j}=\lambda+1}^{\mathrm{A}} \Gamma\left(\mathrm{a}_{\mathrm{j}}-\sum_{\mathrm{i}=1}^{\mathrm{r}} \theta_{\mathrm{j}}^{(\mathrm{i})} \mathrm{s}_{\mathrm{i}}\right) \prod_{\mathrm{j}=1}^{\mathrm{C}} \Gamma\left(1-\mathrm{c}_{j}+\sum_{\mathrm{i}=1}^{\mathrm{r}} \psi_{j}^{(\mathrm{i})} \mathrm{s}_{\mathrm{i}}\right)},
$$

For more details about this function, we may refer to [7].

For the sake of brevity,

$$
\begin{aligned}
& \Delta=\left(\frac{\pi}{2^{2+\sigma}}\right) \bar{H}_{\mathrm{p}, \mathrm{q}}^{\mathrm{m}, \mathrm{n}}\left[\left(\alpha^{\prime}\right)\left(2^{-\mathrm{k}}\right) \mid \begin{array}{c}
\left(\mathrm{a}_{\mathrm{j}}, \alpha_{\mathrm{j}} ; \mathrm{A}_{\mathrm{j}}\right)_{1, \mathrm{n}},\left(\mathrm{a}_{\mathrm{j}}, \alpha_{\mathrm{j}}\right)_{\mathrm{n}+1, \mathrm{p}} \\
\left(\mathrm{b}_{\mathrm{j}}, \beta_{\mathrm{j}}\right)_{1, \mathrm{~m}},\left(\mathrm{~b}_{\mathrm{j}}, \beta_{\mathrm{j}} ; \mathrm{B}_{\mathrm{j}}\right)_{\mathrm{m}+1, \mathrm{q}}
\end{array}\right] . \\
& \nabla=\frac{\ell_{\mathrm{d}-1} \Gamma(\mu+1) \Gamma\left(\frac{1}{2}+\frac{\mathfrak{J}}{2}\right)}{(-1)^{\mu} 2^{2+\mu}(\pi)^{\frac{1}{2}} \Gamma(\gamma) \Gamma\left(\gamma-\frac{\mathfrak{I}}{2}\right)}\left(\frac{\pi}{2^{2+\sigma}}\right) \\
& \times \overline{\mathrm{H}}_{3,3}^{1,3}\left[\begin{array}{l|l}
\left(-\alpha^{\prime}\right)\left(2^{-\mathrm{k}}\right) & \begin{array}{l}
\left.\left(\mathrm{a}_{\mathrm{j}}, \alpha_{\mathrm{j}} ; \mathrm{A}_{\mathrm{j}}\right)_{1, \mathrm{n}},\left(\mathrm{a}_{\mathrm{j}}, \alpha_{\mathrm{j}}\right)_{\mathrm{n}+1, \mathrm{p}},(1-\gamma, 1 ; 1),\left(1-\gamma+\frac{\mathfrak{I}}{2}, 1 ; 1\right),(1-\eta, 1 ; 1+\mu)\right) \\
(0,1),\left(-\frac{\mathfrak{J}}{2}, 1 ; 1\right),(-\eta, 1 ; 1),(-\eta, 1 ; 1+\mu),\left(\mathrm{b}_{\mathrm{j}}, \beta_{\mathrm{j}}\right)_{1, \mathrm{~m}},\left(\mathrm{~b}_{\mathrm{j}}, \beta_{\mathrm{j}} ; \mathrm{B}_{\mathrm{j}}\right)_{\mathrm{m}+1, \mathrm{q}}
\end{array}
\end{array}\right] \\
& \xi=\left(\frac{\pi}{2^{2+\sigma}}\right) \mathrm{H}_{\mathrm{p}, \mathrm{q}}^{\mathrm{m}, \mathrm{q}}\left[\left(\alpha^{\prime}\right)\left(2^{-\mathrm{k}}\right) \mid \begin{array}{l}
\left(\mathrm{a}_{\mathrm{j}}, \alpha_{\mathrm{j}}\right)_{1, \mathrm{p}} \\
\left(\mathrm{b}_{\mathrm{j}}, \beta_{\mathrm{j}}\right)_{1, \mathrm{q}}
\end{array}\right] \text {. }
\end{aligned}
$$

\section{The Main Triple Integral Relations}

Our main results of the present paper are the triple integral relations contained in the following Theorems.

\section{THEOREM 1}

$$
\left.\begin{array}{l}
\int_{0}^{\infty} \int_{0}^{\infty} \int_{0}^{\infty} \frac{(\mathrm{tu})^{\sigma}}{\left(\mathrm{t}^{2}+\mathrm{u}^{2}+\mathrm{z}^{2}\right)^{\sigma}} \cos \left(2 \delta \tan ^{-1} \frac{\mathrm{z}}{\mathrm{t}}\right) \\
\overline{\mathrm{H}}_{\mathrm{p}, \mathrm{q}}^{\mathrm{m}, \mathrm{n}}\left[\frac{\left(\alpha^{\prime}\right)(\mathrm{tu})^{\mathrm{k}}}{\left(\mathrm{t}^{2}+\mathrm{u}^{2}+\mathrm{z}^{2}\right)^{\mathrm{k}}} \mid \begin{array}{c}
\left(\mathrm{a}_{\mathrm{j}}, \mathrm{\alpha}_{\mathrm{j}} ; \mathrm{A}_{\mathrm{j}}\right)_{1, \mathrm{n}},\left(\mathrm{a}_{\mathrm{j}}, \alpha_{\mathrm{j}}\right)_{\mathrm{n}+1, \mathrm{p}} \\
\left(\mathrm{b}_{\mathrm{j}}, \beta_{\mathrm{j}}\right)_{1, \mathrm{~m}},\left(\mathrm{~b}_{\mathrm{j}}, \beta_{\mathrm{j}} ; \mathrm{B}_{\mathrm{j}}\right)_{\mathrm{m}+1, \mathrm{q}}
\end{array}\right] \\
\mathrm{H}_{\left.\mathrm{A}, \mathrm{C}:\left[\mathrm{B}^{\prime}, \mathrm{D}\right] ; \ldots ; \mathrm{B}^{(\mathrm{r})}, \mathrm{D}^{(\mathrm{r})}\right]}^{0, \lambda:\left(\mathrm{U}^{\prime}, \mathrm{V}^{\prime}\right) ; \ldots ;\left(\mathrm{U}^{(\mathrm{r})}, \mathrm{V}^{(\mathrm{r})}\right)}\left[\begin{array}{c}
\mathrm{y}_{1}(\mathrm{tu})^{\sigma_{1}}\left(\mathrm{t}^{2}+\mathrm{u}^{2}+\mathrm{z}^{2}\right)^{\mathrm{b}_{1}-\sigma_{1}} \\
\mathrm{y}_{2}\left(\mathrm{t}^{2}+\mathrm{u}^{2}+\mathrm{z}^{2}\right)^{\mathrm{b}_{2}} \\
\vdots \\
\mathrm{y}_{\mathrm{r}}\left(\mathrm{t}^{2}+\mathrm{u}^{2}+\mathrm{z}^{2}\right)^{\mathrm{b}_{\mathrm{r}}}
\end{array}\right] \mathrm{f}\left(\mathrm{t}^{2}+\mathrm{u}^{2}+\mathrm{z}^{2}\right) \mathrm{dt} \mathrm{dudz}
\end{array}\right]
$$




$$
\left.\begin{array}{l}
=\Delta \int_{0}^{\infty} \mathrm{H}_{\mathrm{A}, \mathrm{C}:\left[\mathrm{B}^{\prime}+3, \mathrm{~V}^{\prime}+2\right] *}^{0, \lambda}\left[\begin{array}{c}
\mathrm{y}_{1} \rho^{2 \mathrm{~b}_{1}} 2^{-\sigma_{1}} \\
\mathrm{y}_{2} \rho^{2 \mathrm{~b}_{2}} \\
\vdots \\
\mathrm{y}_{\mathrm{r}} \rho^{2 \mathrm{~b}_{\mathrm{r}}}
\end{array} \mid\left[(\mathrm{a}): \theta^{\prime}, \ldots, \theta^{(\mathrm{r})}\right]:\right. \\
{\left[(\mathrm{c}): \psi^{\prime}, \ldots, \psi^{\mathrm{r}}\right]:} \\
\left.\left[\frac{1}{2}-\frac{\sigma}{2}-\frac{\mathrm{ks}}{2} ; \frac{\mathrm{s}}{2}\right]\left[-\frac{\sigma}{2}-\frac{\mathrm{ks}}{2} ; \frac{\mathrm{s}_{1}}{2}\right]\left[-\sigma-\mathrm{ks} ; \mathrm{s}_{1}\right] *\right] \times \rho^{2} \mathrm{f}\left(\rho^{2}\right) \mathrm{d} \rho, \\
{\left[-\frac{1}{2}-\sigma-\mathrm{ks} ; \mathrm{s}_{1}\right]\left[-\frac{\sigma}{2}-\frac{\mathrm{ks}}{2} \pm \delta ; \frac{\mathrm{s}_{1}}{2}\right] *}
\end{array}\right]
$$

where

$$
\begin{aligned}
& {\left[\operatorname{Re}(\sigma)+k s \min \operatorname{Re}\left(\frac{b_{j}}{\beta_{j}}\right)-\sum_{i=1}^{r} b_{i} \sigma_{i} \max \operatorname{Re}\left(\frac{b_{j}^{(i)}-1}{\phi_{j}^{(i)}}\right)+\sigma_{1} s_{1} \min \operatorname{Re}\left(\frac{d_{j}^{\prime}}{\delta_{j}^{\prime}}\right)-\frac{1}{2}\right]>0} \\
& {\left[\operatorname{Re}(\sigma)+k \min \operatorname{Re}\left(\frac{b_{j}}{\beta_{j}}\right)+\sigma_{1} s_{1} \min \left(\frac{d_{j}^{\prime}}{\delta_{j}^{\prime}}\right)+1\right)>0} \\
& {\left[\operatorname{Re}(\sigma)+k s \min \operatorname{Re}\left(\frac{b_{j}}{\beta_{j}}\right)-2 \sum_{i=1}^{r} b_{i} \sigma_{i} \max \operatorname{Re}\left(\frac{b_{j}^{(i)}-1}{\phi_{j}^{(i)}}\right)+\sigma_{1} s_{1} \min \operatorname{Re}\left(\frac{d_{j}^{\prime}}{\delta_{j}^{\prime}}\right)-1\right]>0,}
\end{aligned}
$$

where the asterisk * in (2.1) indicates that the parameters at these places are the same as the parameters of the $\mathrm{H}$-function of several variables defined by (1.3)

\section{THEOREM 2}

$$
\left.\begin{array}{l}
\int_{0}^{\infty} \int_{0}^{\infty} \int_{0}^{\infty} \frac{(\mathrm{tu})^{\sigma}}{\left(\mathrm{t}^{2}+\mathrm{u}^{2}+\mathrm{z}^{2}\right)^{\sigma}} \cos \left(2 \delta \tan ^{-1} \frac{\mathrm{z}}{\mathrm{t}}\right) \\
\overline{\mathrm{H}}_{\mathrm{p}, \mathrm{q}}^{\mathrm{m}, \mathrm{n}}\left[\frac{\left(\alpha^{\prime}\right)(\mathrm{tu})^{\mathrm{k}}}{\left(\mathrm{t}^{2}+\mathrm{u}^{2}+\mathrm{z}^{2}\right)^{\mathrm{k}}} \mid \begin{array}{c}
\left(\mathrm{a}_{\mathrm{j}}, \alpha_{\mathrm{j}} ; \mathrm{A}_{\mathrm{j}}\right)_{1, \mathrm{n}},\left(\mathrm{a}_{\mathrm{j}}, \alpha_{\mathrm{j}}\right)_{\mathrm{n}+1, \mathrm{p}} \\
\left.\left(\mathrm{b}_{\mathrm{j}}, \beta_{\mathrm{j}}\right)_{1, \mathrm{~m}}, \mathrm{~b}_{\mathrm{j}}, \beta_{\mathrm{j}} ; \mathrm{B}_{\mathrm{j}}\right)_{\mathrm{m}+1, \mathrm{q}}
\end{array}\right] \\
\mathrm{H}_{\left.\mathrm{A}, \mathrm{C}:\left[\mathrm{B}^{\prime}, \mathrm{D}\right] ; \ldots ; \mathrm{B}^{(\mathrm{r})}, \mathrm{D}^{(\mathrm{r})}\right)}^{0, \lambda:\left(\mathrm{U}^{\prime}, \mathrm{V}^{\prime}\right) ; \ldots ;\left(\mathrm{U}^{(\mathrm{r})}, \mathrm{V}^{(\mathrm{r})}\right)}\left[\begin{array}{c}
\mathrm{y}_{1}(\mathrm{tu})^{-\sigma_{1}}\left(\mathrm{t}^{2}+\mathrm{u}^{2}+\mathrm{z}^{2}\right)^{\mathrm{b}_{1}+\sigma_{1}} \\
\mathrm{y}_{2}\left(\mathrm{t}^{2}+\mathrm{u}^{2}+\mathrm{z}^{2}\right)^{\mathrm{b}_{2}} \\
\vdots \\
\mathrm{y}_{\mathrm{r}}\left(\mathrm{t}^{2}+\mathrm{u}^{2}+\mathrm{z}^{2}\right)^{\mathrm{b}_{\mathrm{r}}}
\end{array}\right] \mathrm{f}\left(\mathrm{t}^{2}+\mathrm{u}^{2}+\mathrm{z}^{2}\right) \mathrm{dtdudz}
\end{array}\right]
$$




$$
\begin{aligned}
& =\Delta \int_{0}^{\infty} \mathrm{H}_{\mathrm{A}, \mathrm{C}:\left[\mathrm{B}^{\prime}+2, \mathrm{D}^{\prime}+3\right] *}^{0, \lambda:\left(\mathrm{J}^{\prime}+3, \mathrm{~V}\right) *}\left[\begin{array}{c}
\mathrm{y}_{1} \rho^{2 \mathrm{~b}_{1}} 2^{\sigma_{1}} \\
\mathrm{y}_{2} \rho^{2 \mathrm{~b}_{2}} \\
\vdots \\
\mathrm{y}_{\mathrm{r}} \rho^{2 \mathrm{~b}_{\mathrm{r}}}
\end{array} \mid\left[(\mathrm{a}): \theta^{\prime}, \ldots, \theta^{(\mathrm{r})}\right]:\right. \\
& \left.\left[1+\frac{\sigma}{2}+\frac{\mathrm{ks}}{2} ; \frac{\mathrm{s}}{2}\right]\left[\frac{1}{2}+\frac{\sigma}{2}-\frac{\mathrm{ks}}{2} ; \frac{\mathrm{s}}{2}\right]\left[1+\sigma+\mathrm{ks} ; \mathrm{s}_{1}\right] *\right] \\
& {\left[\frac{3}{2}+\sigma+\mathrm{ks} ; \mathrm{s}_{1}\right]\left[1+\frac{\sigma}{2}+\frac{\mathrm{ks}}{2} \pm \delta ; \frac{1}{2}\right] *} \\
& \times \rho^{2} f\left(\rho^{2}\right) d \rho,
\end{aligned}
$$

valid under the conditions as obtainable from (2.1).

\section{THEOREM 3}

$$
\begin{aligned}
& \int_{0}^{\infty} \int_{0}^{\infty} \int_{0}^{\infty} \frac{(\mathrm{tu})^{\sigma}}{\left(\mathrm{t}^{2}+\mathrm{u}^{2}+\mathrm{z}^{2}\right)^{\sigma}} \cos \left(2 \delta \tan ^{-1} \frac{\mathrm{z}}{\mathrm{t}}\right) \\
& \bar{H}_{p, q}^{m, n}\left[\frac{\left(\alpha^{\prime}\right)(t u)^{k}}{\left(t^{2}+u^{2}+z^{2}\right)^{k}} \mid \begin{array}{l}
\left(a_{j}, \alpha_{j} ; A_{j}\right)_{1, n},\left(a_{j}, \alpha_{j}\right)_{n+1, p} \\
\left(b_{j}, \beta_{j}\right)_{1, m},\left(b_{j}, \beta_{j} ; B_{j}\right)_{m+1, q}
\end{array}\right] \\
& \mathrm{H}_{\left.\mathrm{A}, \mathrm{C}:\left[\mathrm{B}^{\prime}, \mathrm{D}\right] ; \ldots ; \mathrm{B}^{(\mathrm{r})}, \mathrm{D}^{(\mathrm{r})}\right]}^{0, \lambda:\left(\mathrm{U}^{\prime}, \mathrm{V}^{\prime}\right) ; \ldots ;\left(\mathrm{U}^{(\mathrm{r})}, \mathrm{V}^{(\mathrm{r})}\right)}\left[\begin{array}{c}
\mathrm{y}_{1}(\mathrm{tu})^{\sigma_{1}}\left(\mathrm{t}^{2}+\mathrm{u}^{2}+\mathrm{z}^{2}\right)^{\mathrm{b}_{1}-\sigma_{1}} \\
\mathrm{y}_{2}(\mathrm{tu})^{\sigma_{2}}\left(\mathrm{t}^{2}+\mathrm{u}^{2}+\mathrm{z}^{2}\right)^{\mathrm{b}_{2}-\sigma_{2}} \\
\vdots \\
\mathrm{y}_{\mathrm{r}}(\mathrm{tu})^{\sigma_{\mathrm{r}}}\left(\mathrm{t}^{2}+\mathrm{u}^{2}+\mathrm{z}^{2}\right)^{\mathrm{b}_{\mathrm{r}}-\sigma_{\mathrm{r}}}
\end{array}\right] \mathrm{f}\left(\mathrm{t}^{2}+\mathrm{u}^{2}+\mathrm{z}^{2}\right) \mathrm{dt} \mathrm{dudz} \\
& =\Delta \int_{0}^{\infty} H_{\mathrm{A}+3, \mathrm{C}+2 *}^{0, \lambda+3 *}\left[\begin{array}{c}
\mathrm{y}_{1} \rho^{2 \mathrm{~b}_{1}} 2^{-\sigma_{1}} \\
\mathrm{y}_{2} \rho^{2 \mathrm{~b}_{2}} 2^{-\sigma_{2}} \\
\vdots \\
\mathrm{y}_{\mathrm{r}} \rho^{2 \mathrm{~b}_{\mathrm{r}}} 2^{-\sigma_{\mathrm{r}}}
\end{array} \mid\left[(\mathrm{a}): \theta^{\prime}, \ldots, \theta^{(\mathrm{r})}\right]:\right. \\
& \left.\left[-\frac{\sigma}{2}-\frac{\mathrm{ks}}{2} ; \frac{\mathrm{s}_{1}}{2}\right]\left[\frac{1}{2}-\frac{\sigma}{2}-\frac{\mathrm{ks}}{2} ; \frac{\mathrm{s}_{1}}{2}\right]\left[-\sigma-\mathrm{ks}_{\mathrm{s}} \mathrm{s}_{1}\right] *\right] \\
& {\left[-\frac{1}{2}-\sigma-\mathrm{ks} ; \mathrm{s}_{1}\right]\left[-\frac{\sigma}{2}-\frac{\mathrm{ks}}{2} \pm \delta ; \frac{\mathrm{s}_{1}}{2}\right] *} \\
& \times \rho^{2} f\left(\rho^{2}\right) d \rho .
\end{aligned}
$$

where the condition of validity are same as surrounding Theorem 1 . 
Proof. To prove Theorem 1, first we change the left hand side of integral (2.1) from Cartesian to Polar form and then expressing the $\overline{\mathrm{H}}$-function and the multivariable $\mathrm{H}$-function in their contour form with the help of (1.1) and (1.3). Now changing the order of integration, we get the following form of integral say $(\chi)$

$$
\begin{aligned}
& \chi=\frac{1}{(2 \pi \omega)^{\mathrm{r}}} \int_{\mathrm{L}_{1}} \ldots \int_{\mathrm{L}_{\mathrm{r}}} \mathrm{T}\left(\mathrm{s}_{1}, \ldots, \mathrm{s}_{\mathrm{r}}\right) \mathrm{R}_{1}\left(\mathrm{~s}_{1}\right) \ldots \mathrm{R}_{\mathrm{r}}\left(\mathrm{s}_{\mathrm{r}}\right) \mathrm{y}_{1}^{\mathrm{s}_{1} \ldots \mathrm{y}_{\mathrm{r}}^{\mathrm{s}_{\mathrm{r}}}} \\
& \frac{1}{(2 \pi \omega)} \int_{\mathrm{L}} \theta(\mathrm{s})\left(\alpha^{\prime}\right)^{\mathrm{s}}\left[\int_{0}^{\infty} \int_{0}^{\pi / 2} \rho^{2+2 \mathrm{~b}_{1} \mathrm{~s}_{1}+\ldots+2 \mathrm{~b}_{\mathrm{r}} \mathrm{s}_{\mathrm{r}}} \mathrm{f}\left(\rho^{2}\right)(\sin \theta)^{\sigma+\mathrm{ks}+\sigma_{1} \mathrm{~s}_{1}+1}\right. \\
& \left.(\cos \theta)^{\sigma+\mathrm{ks}+\sigma_{1} \mathrm{~s}_{1}}\left\{\int_{0}^{\pi / 2}(\cos \phi)^{\sigma+\mathrm{ks}+\sigma_{1} \mathrm{~s}_{1}} \cos (2 \delta \phi) \mathrm{d} \phi\right\} \mathrm{d} \theta \mathrm{d} \rho\right] \mathrm{ds} \cdot \mathrm{d} \mathrm{s}_{1} \ldots \mathrm{d} \mathrm{s}_{\mathrm{r}} .
\end{aligned}
$$

On evaluating the $\theta$ and $\phi$ integral occurring on the right hand side of (2.4) with the help of known result [3, Eq.5, p.16], see also [4, Eq.3.2.7, p.62] and using the well known Beta function, we easily arrive at the desired result (2.1) after a little simplification.

Theorems 2 and 3 can be proved on the similar way.

\section{Special Cases}

(I) Taking $\mathrm{m}=1, \mathrm{n}=3=\mathrm{p}=\mathrm{q}$ and replacing $\mathrm{z}$ by $-\mathrm{z}$ in the triple integral (2.1) through (2.3) and using

$$
\begin{aligned}
& \mathrm{g}(\gamma, \eta, \mathfrak{I}, \mu ; \mathrm{z})=\frac{\ell_{\mathrm{d}-1} \Gamma(\mu+1) \Gamma\left(\frac{1}{2}+\frac{\mathfrak{I}}{2}\right)}{(-1)^{\mu} 2^{2+\mu}(\pi)^{\frac{1}{2}} \Gamma(\gamma) \Gamma\left(\gamma-\frac{\mathfrak{I}}{2}\right)} \\
& \times \overline{\mathrm{H}}_{3,3}^{1,3}\left[-\mathrm{z} \mid \begin{array}{l}
(1-\gamma, 1 ; 1),\left(1-\gamma+\frac{\mathfrak{I}}{2}, 1 ; 1\right),(1-\eta, 1 ; 1+\mu) \\
(0,1),\left(-\frac{\mathfrak{J}}{2}, 1 ; 1\right),(-\eta, 1 ; 1+\mu)
\end{array}\right],
\end{aligned}
$$

where $\ell_{\mathrm{d}}=\frac{2^{1-\mathrm{d}}(\pi)^{-\frac{\mathrm{d}}{2}}}{\Gamma\left(\frac{\mathrm{d}}{2}\right)}([2]$, p.4121, Eqn. (5)).

The above function is connected with a certain class of Feynman integrals. We get

\section{THEOREM 4}

$$
\begin{aligned}
& \int_{0}^{\infty} \int_{0}^{\infty} \int_{0}^{\infty} \frac{(\mathrm{tu})^{\sigma}}{\left(\mathrm{t}^{2}+\mathrm{u}^{2}+\mathrm{z}^{2}\right)^{\sigma}} \cos \left(2 \delta \tan ^{-1} \frac{\mathrm{z}}{\mathrm{t}}\right) \\
& \mathrm{g}\left(\gamma, \eta, \mathfrak{J}, \mu ; \frac{(\alpha)^{\prime}(\mathrm{tu})^{\mathrm{k}}}{\left(\mathrm{t}^{2}+\mathrm{u}^{2}+\mathrm{z}^{2}\right)^{\mathrm{k}}}\right) \\
& \mathrm{H}_{\mathrm{A}, \mathrm{C}:\left[\mathrm{B}^{\prime}, \mathrm{D}\right] ; ; \ldots ;\left[\mathrm{B}^{(\mathrm{r})}, \mathrm{D}^{(\mathrm{r})}\right]}^{0, \lambda:\left(\mathrm{U}^{\prime}, \mathrm{V}^{\prime}\right) ; \ldots ;\left(\mathrm{U}^{(\mathrm{r})}, \mathrm{V}^{(\mathrm{r})}\right)}\left[\begin{array}{c}
\mathrm{y}_{1}(\mathrm{tu})^{\sigma_{1}}\left(\mathrm{t}^{2}+\mathrm{u}^{2}+\mathrm{z}^{2}\right)^{\mathrm{b}_{1}-\sigma_{1}} \\
\mathrm{y}_{2}\left(\mathrm{t}^{2}+\mathrm{u}^{2}+\mathrm{z}^{2}\right)^{\mathrm{b}_{2}} \\
\vdots \\
\mathrm{y}_{\mathrm{r}}\left(\mathrm{t}^{2}+\mathrm{u}^{2}+\mathrm{z}^{2}\right)^{\mathrm{b}_{\mathrm{r}}}
\end{array}\right] \mathrm{f}\left(\mathrm{t}^{2}+\mathrm{u}^{2}+\mathrm{z}^{2}\right) \mathrm{dt} \mathrm{dudz}
\end{aligned}
$$




$$
\begin{aligned}
& =\nabla \int_{0}^{\infty} \mathrm{H}_{\mathrm{A}, \mathrm{C}:\left[\mathrm{B}^{\prime}+3, \mathrm{D}^{\prime}+2\right] *}^{0, \lambda:\left(\mathrm{U}^{\prime} \mathrm{V}^{\prime}+3\right) *}\left[\begin{array}{c}
\mathrm{y}_{1} \rho^{2 \mathrm{~b}_{1}} 2^{-\sigma_{1}} \\
\mathrm{y}_{2} \rho^{2 \mathrm{~b}_{2}} \\
\vdots \\
\mathrm{y}_{\mathrm{r}} \rho^{2 \mathrm{~b}_{\mathrm{r}}}
\end{array} \mid\left[(\mathrm{a}): \theta^{\prime}, \ldots, \theta^{(\mathrm{r})}\right]:\right. \\
& \left.\left[-\frac{\sigma}{2}-\frac{\mathrm{ks}}{2} ; \frac{\mathrm{s}}{2}\right]\left[\frac{1}{2}-\frac{\sigma}{2}-\frac{\mathrm{ks}}{2} ; \frac{\mathrm{s}}{2}\right]\left[-\sigma-\mathrm{ks} ; \mathrm{s}_{1}\right] *\right] \\
& {\left[-\frac{1}{2}-\sigma-\mathrm{ks} ; \mathrm{s}_{1}\right]\left[-\frac{\sigma}{2}-\frac{\mathrm{ks}}{2} \pm \delta ; \frac{\mathrm{s}_{1}}{2}\right] * \rho^{2} \mathrm{f}\left(\rho^{2}\right) \mathrm{d} \rho .}
\end{aligned}
$$

valid under conditions as obtainable from (2.1)

\section{THEOREM 5}

$$
\begin{aligned}
& \int_{0}^{\infty} \int_{0}^{\infty} \int_{0}^{\infty} \frac{(\mathrm{tu})^{\sigma}}{\left(\mathrm{t}^{2}+\mathrm{u}^{2}+\mathrm{z}^{2}\right)^{\sigma}} \cos \left(2 \delta \tan ^{-1} \frac{\mathrm{z}}{\mathrm{t}}\right) \\
& \mathrm{g}\left(\gamma, \eta, \mathfrak{J}, \mu ; \frac{(\alpha)^{\prime}(\mathrm{tu})^{\mathrm{k}}}{\left(\mathrm{t}^{2}+\mathrm{u}^{2}+\mathrm{z}^{2}\right)^{\mathrm{k}}}\right) \\
& \mathrm{H}_{\mathrm{A}, \mathrm{C}:\left[\mathrm{B}^{\prime}, \mathrm{D}\right] ; \ldots ;\left[\mathrm{B}^{(\mathrm{r})}, \mathrm{D}^{(\mathrm{r})}\right]}^{0, \lambda:\left(\mathrm{U}^{\prime}, \mathrm{V}\right) ; \ldots ;\left(\mathrm{U}^{(\mathrm{r})}, \mathrm{V}^{(\mathrm{r})}\right)}\left[\begin{array}{c}
\mathrm{y}_{1}(\mathrm{tu})^{-\sigma_{1}}\left(\mathrm{t}^{2}+\mathrm{u}^{2}+\mathrm{z}^{2}\right)^{\mathrm{b}_{1}+\sigma_{1}} \\
\mathrm{y}_{2}\left(\mathrm{t}^{2}+\mathrm{u}^{2}+\mathrm{z}^{2}\right)^{\mathrm{b}_{2}} \\
\vdots \\
\mathrm{y}_{\mathrm{r}}\left(\mathrm{t}^{2}+\mathrm{u}^{2}+\mathrm{z}^{2}\right)^{\mathrm{b}_{\mathrm{r}}}
\end{array}\right] \mathrm{f}\left(\mathrm{t}^{2}+\mathrm{u}^{2}+\mathrm{z}^{2}\right) \mathrm{dt} \mathrm{dudz} \\
& =\nabla \int_{0}^{\infty} \mathrm{H}_{\mathrm{A}, \mathrm{C}:\left[\mathrm{B}^{\prime}+2, \mathrm{D}^{\prime}+3\right] *}^{0, \lambda:\left(\mathrm{U}^{\prime}+3, \mathrm{~V}^{\prime}\right) *}\left[\begin{array}{c}
\mathrm{y}_{1} \rho^{2 \mathrm{~b}_{1}} 2^{\sigma_{1}} \\
\mathrm{y}_{2} \rho^{2 \mathrm{~b}_{2}} \\
\vdots \\
\mathrm{y}_{\mathrm{r}} \rho^{2 \mathrm{~b}_{\mathrm{r}}}
\end{array} \mid\left[(\mathrm{a}): \theta^{\prime}, \ldots, \theta^{(\mathrm{r})}\right]:\right. \\
& \left.\left[1+\frac{\sigma}{2}+\frac{\mathrm{ks}}{2} ; \frac{\mathrm{s}_{1}}{2}\right]\left[\frac{1}{2}+\frac{\sigma}{2}+\frac{\mathrm{ks}}{2} ; \frac{\mathrm{s}_{1}}{2}\right]\left[1+\sigma+\mathrm{ks} ; \mathrm{s}_{1}\right] *\right] \\
& {\left[\frac{3}{2}+\sigma+\mathrm{ks}^{2} \mathrm{~s}_{1}\right]\left[1+\frac{\sigma}{2}+\frac{\mathrm{ks}}{2} \pm \delta ; \frac{\mathrm{s}_{1}}{2}\right] *} \\
& \times \rho^{2} f\left(\rho^{2}\right) d \rho .
\end{aligned}
$$

valid under the conditions as needed for (2.2). 


\section{THEOREM 6}

$$
\begin{aligned}
& \int_{0}^{\infty} \int_{0}^{\infty} \int_{0}^{\infty} \frac{(\mathrm{tu})^{\sigma}}{\left(\mathrm{t}^{2}+\mathrm{u}^{2}+\mathrm{z}^{2}\right)^{\sigma}} \cos \left(2 \delta \tan ^{-1} \frac{\mathrm{z}}{\mathrm{t}}\right) \\
& \mathrm{g}\left(\gamma, \eta, \mathfrak{J}, \mu ; \frac{(\alpha)^{\prime}(\mathrm{tu})^{\mathrm{k}}}{\left(\mathrm{t}^{2}+\mathrm{u}^{2}+\mathrm{z}^{2}\right)^{\mathrm{k}}}\right) \\
& \mathrm{H}_{\left.\mathrm{A}, \mathrm{C}:\left[\mathrm{B}^{\prime}, \mathrm{D}\right] ; \ldots ; \mathrm{B}^{(\mathrm{r})}, \mathrm{D}^{(\mathrm{r})}\right]}^{0, \lambda:\left(\mathrm{U}^{\prime}, \mathrm{V}^{\prime}\right) ; \ldots ;\left(\mathrm{U}^{(\mathrm{r})}, \mathrm{V}^{(\mathrm{r})}\right)}\left[\begin{array}{c}
\mathrm{y}_{1}(\mathrm{tu})^{\sigma_{1}}\left(\mathrm{t}^{2}+\mathrm{u}^{2}+\mathrm{z}^{2}\right)^{\mathrm{b}_{1}-\sigma_{1}} \\
\mathrm{y}_{2}(\mathrm{tu})^{\sigma_{2}}\left(\mathrm{t}^{2}+\mathrm{u}^{2}+\mathrm{z}^{2}\right)^{\mathrm{b}_{2}-\sigma_{2}} \\
\vdots \\
\mathrm{y}_{\mathrm{r}}(\mathrm{tu})^{\sigma_{\mathrm{r}}}\left(\mathrm{t}^{2}+\mathrm{u}^{2}+\mathrm{z}^{2}\right)^{\mathrm{b}_{\mathrm{r}}-\sigma_{\mathrm{r}}}
\end{array}\right] \mathrm{f}\left(\mathrm{t}^{2}+\mathrm{u}^{2}+\mathrm{z}^{2}\right) \mathrm{dtdudz} \\
& =\nabla \int_{0}^{\infty} \mathrm{H}_{\mathrm{A}+3, \mathrm{C}+2: *}^{0, \lambda+3: *}\left[\begin{array}{c}
\mathrm{y}_{1} \rho^{2 \mathrm{~b}_{1}} 2^{-\sigma_{1}} \\
\mathrm{y}_{2} \rho^{2 \mathrm{~b}_{2}} 2^{-\sigma_{2}} \\
\vdots \\
\mathrm{y}_{\mathrm{r}} \rho^{2 \mathrm{~b}_{\mathrm{r}}} 2^{-\sigma_{\mathrm{r}}}
\end{array} \mid\left[(\mathrm{a}): \theta^{\prime}, \ldots, \theta^{(\mathrm{r})}\right]:\right. \\
& \left.\left[-\frac{\sigma}{2}-\frac{\mathrm{ks}}{2} ; \frac{\mathrm{s}_{1}}{2}\right]\left[\frac{1}{2}-\frac{\sigma}{2}-\frac{\mathrm{ks}}{2} ; \frac{\mathrm{s}_{1}}{2}\right]\left[-\sigma-\mathrm{ks} ; \mathrm{s}_{1}\right] *\right] \\
& {\left[-\frac{1}{2}-\sigma-\mathrm{ks}_{\mathrm{s}} \mathrm{s}_{1}\right]\left[-\frac{\sigma}{2}-\frac{\mathrm{ks}}{2} \pm \delta ; \frac{\mathrm{s}_{1}}{2}\right] *} \\
& \times \rho^{2} f\left(\rho^{2}\right) d \rho .
\end{aligned}
$$

(II) Putting $\mathrm{A}_{\mathrm{j}}=\mathrm{B}_{\mathrm{j}}=1, \forall \mathrm{j}$ the results from (2.1) to (2.3) reduce to a known result derived by Chaurasia and Saxena [1].

\section{THEOREM 7}

$$
\left.\begin{array}{l}
\int_{0}^{\infty} \int_{0}^{\infty} \int_{0}^{\infty} \frac{(\mathrm{tu})^{\sigma}}{\left(\mathrm{t}^{2}+\mathrm{u}^{2}+\mathrm{z}^{2}\right)^{\sigma}} \cos \left(2 \delta \tan ^{-1} \frac{\mathrm{z}}{\mathrm{t}}\right) \\
\mathrm{H}_{\mathrm{p}, \mathrm{q}}^{\mathrm{m}, \mathrm{n}}\left[\frac{\left(\alpha^{\prime}\right)(\mathrm{tu})^{\mathrm{k}}}{\left(\mathrm{t}^{2}+\mathrm{u}^{2}+\mathrm{z}^{2}\right)^{\mathrm{k}}} \mid \begin{array}{l}
\left(\mathrm{a}_{\mathrm{j}}, \alpha_{\mathrm{j}}\right)_{1, \mathrm{p}} \\
\left(\mathrm{b}_{\mathrm{j}}, \beta_{\mathrm{j}}\right)_{1, \mathrm{q}}
\end{array}\right] \\
\left.\mathrm{H}_{\left.\mathrm{A}, \mathrm{C}:\left[\mathrm{B}^{\prime}, \mathrm{D}^{\prime}\right] ; \ldots ; \mathrm{B}^{(\mathrm{r})}, \mathrm{D}^{(\mathrm{r})}\right]}^{0, \lambda:\left(\mathrm{U}^{\prime}, \mathrm{V}^{\prime}\right) ; \ldots ;\left(\mathrm{U}^{(\mathrm{r})}, \mathrm{V}^{(\mathrm{r})}\right)}\left[\begin{array}{c}
\mathrm{y}_{1}(\mathrm{tu})^{\sigma_{1}}\left(\mathrm{t}^{2}+\mathrm{u}^{2}+\mathrm{z}^{2}\right)^{\mathrm{b}_{1}-\sigma_{1}} \\
\mathrm{y}_{2}\left(\mathrm{t}^{2}+\mathrm{u}^{2}+\mathrm{z}^{2}\right)^{\mathrm{b}_{2}} \\
\vdots \\
\mathrm{y}_{\mathrm{r}}\left(\mathrm{t}^{2}+\mathrm{u}^{2}+\mathrm{z}^{2}\right)^{\mathrm{b}_{\mathrm{r}}}
\end{array}\right] \mathrm{f}^{2}+\mathrm{u}^{2}+\mathrm{z}^{2}\right) \mathrm{dt} \mathrm{dudz}
\end{array}\right]
$$




$$
\begin{aligned}
& =\xi \int_{0}^{\infty} \mathrm{H}_{\mathrm{A}, \mathrm{C}:\left[\mathrm{B}^{\prime}+3, \mathrm{D}^{\prime}+2\right] *}^{0, \lambda:\left(\mathrm{V}^{\prime}+3\right) *}\left[\begin{array}{c}
\mathrm{y}_{1} \rho^{2 \mathrm{~b}_{1}} 2^{-\sigma_{1}} \\
\mathrm{y}_{2} \rho^{2 \mathrm{~b}_{2}} \\
\vdots \\
\mathrm{y}_{\mathrm{r}} \rho^{2 \mathrm{~b}_{\mathrm{r}}}
\end{array} \mid\left[(\mathrm{a}): \theta^{\prime}, \ldots, \theta^{(\mathrm{r})}\right]:\right. \\
& \left.\left[\frac{1}{2}-\frac{\sigma}{2}-\frac{\mathrm{ks}}{2} ; \frac{\mathrm{s}}{2}\right]\left[-\frac{\sigma}{2}-\frac{\mathrm{ks}}{2} ; \frac{\mathrm{s}}{2}\right]\left[-\sigma-\mathrm{ks} ; \mathrm{s}_{1}\right] *\right] \\
& {\left[-\frac{1}{2}-\sigma-\mathrm{ks} ; \mathrm{s}_{1}\right]\left[-\frac{\sigma}{2}-\frac{\mathrm{ks}}{2} \pm \delta ; \frac{\mathrm{s}_{1}}{2}\right] *}
\end{aligned}
$$

\section{THEOREM 8}

$$
\begin{aligned}
& \int_{0}^{\infty} \int_{0}^{\infty} \int_{0}^{\infty} \frac{(\mathrm{tu})^{\sigma}}{\left(\mathrm{t}^{2}+\mathrm{u}^{2}+\mathrm{z}^{2}\right)^{\sigma}} \cos \left(2 \delta \tan ^{-1} \frac{\mathrm{z}}{\mathrm{t}}\right) \\
& H_{p, q}^{m, n}\left[\frac{\left(\alpha^{\prime}\right)(t u)^{k}}{\left(t^{2}+u^{2}+z^{2}\right)^{k}} \mid \begin{array}{c}
\left(a_{j}, \alpha_{j}\right)_{1, p} \\
\left(b_{j}, \beta_{j}\right)_{1, q}
\end{array}\right] \\
& \mathrm{H}_{\left.\mathrm{A}, \mathrm{C}:\left[\mathrm{B}^{\prime}, \mathrm{D}\right] ; \ldots ; \mathrm{B}^{(\mathrm{r})}, \mathrm{D}^{(\mathrm{r})}\right]}^{0, \lambda:\left(\mathrm{U}^{\prime}, \mathrm{V}^{\prime}\right) ; \ldots ;\left(\mathrm{U}^{(\mathrm{r})}, \mathrm{V}^{(\mathrm{r})}\right)}\left[\begin{array}{c}
\mathrm{y}_{1}(\mathrm{tu})^{-\sigma_{1}}\left(\mathrm{t}^{2}+\mathrm{u}^{2}+\mathrm{z}^{2}\right)^{\mathrm{b}_{1}+\sigma_{1}} \\
\mathrm{y}_{2}\left(\mathrm{t}^{2}+\mathrm{u}^{2}+\mathrm{z}^{2}\right)^{\mathrm{b}_{2}} \\
\vdots \\
\mathrm{y}_{\mathrm{r}}\left(\left(\mathrm{t}^{2}+\mathrm{u}^{2}+\mathrm{z}^{2}\right)^{\mathrm{b}_{\mathrm{r}}}\right.
\end{array}\right] \mathrm{f}\left(\mathrm{t}^{2}+\mathrm{u}^{2}+\mathrm{z}^{2}\right) \mathrm{dtdudz} \\
& =\xi \int_{0}^{\infty} \mathrm{H}_{\mathrm{A}, \mathrm{C}:\left[\mathrm{B}^{\prime}+2, \mathrm{D}^{\prime}+3\right] *}^{0, \lambda:\left(\mathrm{U}^{\prime}+3, \mathrm{~V}\right) *}\left[\begin{array}{c}
\mathrm{y}_{1} \rho^{2 \mathrm{~b}_{1}} 2^{\sigma_{1}} \\
\mathrm{y}_{2} \rho^{2 \mathrm{~b}_{2}} \\
\vdots \\
\mathrm{y}_{\mathrm{r}} \rho^{2 \mathrm{~b}_{\mathrm{r}}}
\end{array} \mid\left[(\mathrm{a}): \theta^{\prime}, \ldots, \theta^{(\mathrm{r})}\right]:\right. \\
& \left.\left[1+\frac{\sigma}{2}+\frac{\mathrm{ks}}{2} ; \frac{\mathrm{s}_{1}}{2}\right]\left[\frac{1}{2}+\frac{\sigma}{2}+\frac{\mathrm{ks}}{2} ; \frac{\mathrm{s}_{1}}{2}\right]\left[1+\sigma+\mathrm{ks} ; \mathrm{s}_{1}\right] *\right] \\
& {\left[\frac{3}{2}+\sigma+\mathrm{ks} ; \mathrm{s}_{1}\right]\left[1+\frac{\sigma}{2}+\frac{\mathrm{ks}}{2} \pm \delta ; \frac{\mathrm{s}_{1}}{2}\right] *} \\
& \times \rho^{2} f\left(\rho^{2}\right) d \rho .
\end{aligned}
$$




\section{THEOREM 9}

$$
\begin{aligned}
& \int_{0}^{\infty} \int_{0}^{\infty} \int_{0}^{\infty} \frac{(\mathrm{tu})^{\sigma}}{\left(\mathrm{t}^{2}+\mathrm{u}^{2}+\mathrm{z}^{2}\right)^{\sigma}} \cos \left(2 \delta \tan ^{-1} \frac{\mathrm{z}}{\mathrm{t}}\right) \\
& H_{p, q}^{m, n}\left[\frac{\left(\alpha^{\prime}\right)(t u)^{k}}{\left(t^{2}+u^{2}+z^{2}\right)^{k}} \mid \begin{array}{c}
\left(a_{j}, \alpha_{j}\right)_{1, p} \\
\left(b_{j}, \beta_{j}\right)_{1, q}
\end{array}\right] \\
& \mathrm{H}_{\left.\mathrm{A}, \mathrm{C}:\left[\mathrm{B}^{\prime}, \mathrm{D}\right] ; \ldots ; \mathrm{B}^{(\mathrm{r})}, \mathrm{D}^{(\mathrm{r})}\right]}^{0, \lambda:\left(\mathrm{U}^{\prime}, \mathrm{V}^{\prime}\right) ; \ldots ;\left(\mathrm{U}^{(\mathrm{r})}, \mathrm{V}^{(\mathrm{r})}\right)}\left[\begin{array}{c}
\mathrm{y}_{1}(\mathrm{tu})^{\sigma_{1}}\left(\mathrm{t}^{2}+\mathrm{u}^{2}+\mathrm{z}^{2}\right)^{\mathrm{b}_{1}-\sigma_{1}} \\
\mathrm{y}_{2}(\mathrm{tu})^{\sigma_{2}}\left(\mathrm{t}^{2}+\mathrm{u}^{2}+\mathrm{z}^{2}\right)^{\mathrm{b}_{2}-\sigma_{2}} \\
\vdots \\
\mathrm{y}_{\mathrm{r}}(\mathrm{tu})^{-\sigma_{\mathrm{r}}}\left(\mathrm{t}^{2}+\mathrm{u}^{2}+\mathrm{z}^{2}\right)^{\mathrm{b}_{\mathrm{r}}-\sigma_{\mathrm{r}}}
\end{array}\right] \mathrm{f}\left(\mathrm{t}^{2}+\mathrm{u}^{2}+\mathrm{z}^{2}\right) \mathrm{dt} \mathrm{dudz} \\
& =\xi \int_{0}^{\infty} H_{A+3, C+2: *}^{0, \lambda+3: *}\left[\begin{array}{c|c}
y_{1} \rho^{2 b_{1}} 2^{-\sigma_{1}} & {\left[(a): \theta^{\prime}, \ldots, \theta^{(r)}\right]:} \\
y_{2} \rho^{2 b_{2}} 2^{-\sigma_{2}} & \vdots \\
y_{r} \rho^{2 b_{r}} 2^{-\sigma_{r}} & {\left[(c): \psi^{\prime}, \ldots, \psi^{r}\right]:}
\end{array}\right. \\
& \left.\left[-\frac{\sigma}{2}-\frac{\mathrm{ks}}{2} ; \frac{\mathrm{s}_{1}}{2}\right]\left[\frac{1}{2}-\frac{\sigma}{2}-\frac{\mathrm{ks}}{2} ; \frac{\mathrm{s}_{1}}{2}\right]\left[-\sigma-\mathrm{ks}_{\mathrm{s}} \mathrm{s}_{1}\right] *\right] \\
& \left.\left[-\frac{1}{2}-\sigma-\mathrm{ks}_{2} \mathrm{~s}_{1}\right]\left[-\frac{\sigma}{2}-\frac{\mathrm{ks}}{2} \pm \delta ; \frac{\mathrm{s}_{1}}{2}\right] *\right]^{\times \rho^{2} \mathrm{f}\left(\rho^{2}\right) \mathrm{d} \rho .}
\end{aligned}
$$

(III) By tacitly giving some values to $\mathrm{f}\left(\rho^{2}\right)$ in (2.1) through (2.3); (3.5) through (3.7) and evaluate $\rho$ integral by means of the known result [7, Eq. 2.4.1, p.15, we can get more triple integral relations.

(IV) A number of other special cases of our results can be obtained by specializing its parameters.

\section{ACKNOWLEDGEMENT}

The authors are grateful to Professor H.M. Srivastava (University of Victoria, Canada) for his kind help and valuable suggestion in the preparation of this paper.

\section{REFERENCES}

[1] V.B.L. Chaurasia and Vishal Saxena, Certain Triple Integral Relations Involving Multivariable H-function : Scientia, Series A: Mathematical Sciences 19 (2010), 69-75.

[2] A.A. Inayat-Hussain, New properties of hypergeometric series derivable from Feynman integrals, II : A generalization of the Hfunction. J. Phys. A : Math. Gen. 20 (1987), 4109-4128.

[3] Y.L. Luke, The Special functions and their approximations, Academic Press, New York and London, I, (1969).

[4] A.M. Mathai and R.K. Saxena; The H-function with Applications, in Statistics and other Disciplines, Wiley Eastern Limited, New Delhi (1978).

[5] H.M. Srivastava and R. Panda, Some bilateral generating functions for a class of generalized hypergeometric polynomials, J. Reine Angew. Math., 283/284, (1976), 265-274. 
[6] H.M. Srivastava and M.C. Daoust, Certain generalized Neumann expansions associatged with the Kampé de Fériet's function, Nederl. Akad. Wetensch. Proc. Ser. A 72, Indag. Math., 31 (1969), 449-457.

[7] H.M. Srivastava, K.C. Gupta and S.P. Goyal, The H-function of One and Two Variables with Applications. South Asian Publishers, New Delhi (1982). 